\title{
PLANCK: Systematic effects induced by periodic fluctuations of arbitrary shape
}

\author{
A. Mennella ${ }^{1}$, M. Bersanelli ${ }^{1,2}$, C. Burigana ${ }^{3}$, D. Maino ${ }^{4}$, N. Mandolesi ${ }^{3}$, \\ G. Morgante ${ }^{3,5}$, and G. Stanghellini ${ }^{3}$
}

1 IFC-CNR, Via Bassini 15, 20133 Milan, Italy

2 Università degli Studi di Milano, Via Celoria 16, 20133 Milan, Italy

3 TESRE-CNR, Via Gobetti 101, Bologna, Italy

4 Osservatorio Astronomico di Trieste, via G. B. Tiepolo 11, 34131 Trieste, Italy

5 Jet Propulsion Laboratories, Pasadena, USA

Received 2 November 2001 / Accepted 4 January 2002

\begin{abstract}
A fundamental requirement in the new generation of high resolution Cosmic Microwave Background imaging experiments is a strict control of systematic errors that must be kept at $\mu \mathrm{K}$ level in the final maps. Some of these errors are of celestial origin, while others will be generated by periodic fluctuations of the satellite environment. These environment instabilities will cause fluctuations in the measured signal output thus generating correlated effects in the reconstructed maps. In this paper we present an analytical study of the impact of periodic signal fluctuations on the measured sky maps produced by the PLANCK survey. In particular we show how it is possible to estimate analytically the damping factor of the peak-to-peak amplitude of the fluctuation at the instrument output after the projection in the final maps.
\end{abstract}

Key words. cosmology: cosmic microwave background, observations - instrumentation: detectors, miscellaneous methods: analytical

\section{Introduction}

The anisotropy pattern in the Cosmic Microwave Background (CMB) radiation carries information about fundamental cosmological properties such as the present expansion rate and average density of the universe, the amount of dark matter, and the nature of the seed fluctuations from which all structures in the universe arose (Vittorio \& Silk 1984; Efstathiou 1987). In 1992, the COBE-DMR maps provided the first experimental evidence of such fluctuations (Smoot et al. 1992) at angular scales $>7^{\circ}$ at a level $\delta T / T \approx 10^{-5}$ (where $T=$ $2.725 \pm 0.002 \mathrm{~K}$, Mather et al. 1999). Since then, several balloon-borne and ground-based experiments have provided CMB anisotropy detection at sub-degree angular scales, thus improving substantially our knowledge of the anisotropy power spectrum. Recently, sub-orbital experiments have been able to map small sky patches with high angular resolution and sensitivity (e.g. Netterfield et al. 2000; De Bernardis et al. 2000; Lee et al. 2001; Stompor et al. 2001; Halverson et al. 2001; Pryke et al. 2001).

Send offprint requests to: A. Mennella, e-mail: daniele@ifctr.mi.cnr.it
Full-sky CMB maps with high resolution and sensitivity, however, can be obtained only by space experiments, where the entire sky is in full view and the environmental conditions are much more favourable (Danese et al. 1996). The NASA satellite MAP (launched in June 2001) will provide the first improved full-sky maps since COBE, while the ESA satellite PLANCK will map the CMB with an accuracy set mainly by astrophysical limits.

A key factor for the success of these missions is a strict control of systematic effects (see, e.g., Bersanelli \& Mandolesi 2000), particularly those that are synchronous with the spacecraft spin period. These fluctuations, in fact, may be particularly difficult to remove from the data stream because of their similarity with the true sky signal, although some data analysis methods have been developed to deal also with spin synchronous signals of celestial origin (Delabrouille et al. 2001).

In many cases the above effects display a periodic behaviour leading to a periodic spurious contribution to the measured signal. While the transfer function from the fluctuation source to the measured signal depends on the details of the instrument and of the effect being considered, the transfer from the measurement output to the sky map does not depend on the source but only on the period of 
the effect and on the scan strategy, and therefore it can be treated independently.

This work is a study of the impact of periodic signal fluctuations on the measured sky maps to be produced by the PLANCK survey. In particular we show how a periodic fluctuation in the instrument output transfers to the final maps and analyse the behaviour of the damping factor versus the oscillation frequency (Sect. 2). In this section we also evaluate the possibility to reduce the level of residual systematic errors by applying destriping algorithms (Sect. 2.2.2), also considering the combination of such systematic errors with instrumental noise. The main result of this analysis is summarised by a general relationship that allows to estimate the final peak-to-peak error in a map starting from a periodic effect of arbitrary shape. In Sect. 3 we discuss the application of this relationship to an example of particular interest for PLANCK-LFI. Finally, in Sect. 4 we draw our main conclusions.

Although the examples presented in this paper are relative to PLANCK, the concepts at the basis of our treatment are general, and can be applied to any CMB imaging experiment.

\section{Transfer of periodic oscillations from instrument output to maps}

\subsection{Level of residual fluctuations in scan circle time-scale}

The PLANCK measurement strategy is such that the signal coming from each resolution element in the sky will be measured approximately 60 times during one scan, thus reducing the effect of fluctuations occurring on time-scales greater than the spin period.

In particular, if we average $N$ measurements of the same pixel taken at times $t_{j}$ then the residual signal oscillation is given by:

$$
\left\langle\delta T_{\mathrm{sky}}(t)\right\rangle_{t_{N}}=\frac{1}{N} \sum_{j=1}^{N} \delta T_{\mathrm{sky}}\left(t_{j}\right)
$$

where $t_{j}=t+(j-1) \tau_{\text {spin }}\left(\tau_{\text {spin }}\right.$ is the spin period which is also the interval between two consecutive measurements of the same pixel in the sky).

Let us consider a sinusoidal signal oscillation with amplitude $A_{\mathrm{f}}$ and period $\tau_{\mathrm{f}}$, i.e. $\delta T_{\text {sky }}(t)=A_{\mathrm{f}} \cos \left(2 \pi t / \tau_{\mathrm{f}}\right)$. Equation (1) takes then the form:

$$
\begin{aligned}
& \left\langle\delta T_{\text {sky }}(t)\right\rangle_{N}=\frac{A_{\mathrm{f}}}{N} \sum_{j=1}^{N} \cos \left(2 \pi t_{j} / \tau_{\mathrm{f}}\right) \\
& =\frac{A_{\mathrm{f}}}{N} \cos \left[\pi\left(2 t+(N-1) \tau_{\text {spin }}\right) / \tau_{\mathrm{f}}\right] \frac{\sin \left(\pi N \tau_{\text {spin }} / \tau_{\mathrm{f}}\right)}{\sin \left(\pi \tau_{\text {spin }} / \tau_{\mathrm{f}}\right)} .
\end{aligned}
$$

Equation (2) gives the amplitude of the signal oscillation after averaging $N$ measurements of the same pixel starting at time $t$. Because the term $\cos \left[\pi\left(2 t+(N-1) \tau_{\text {spin }}\right) / \tau_{\mathrm{f}}\right]$ is bounded by \pm 1 , then the peak-to-peak fluctuation in the

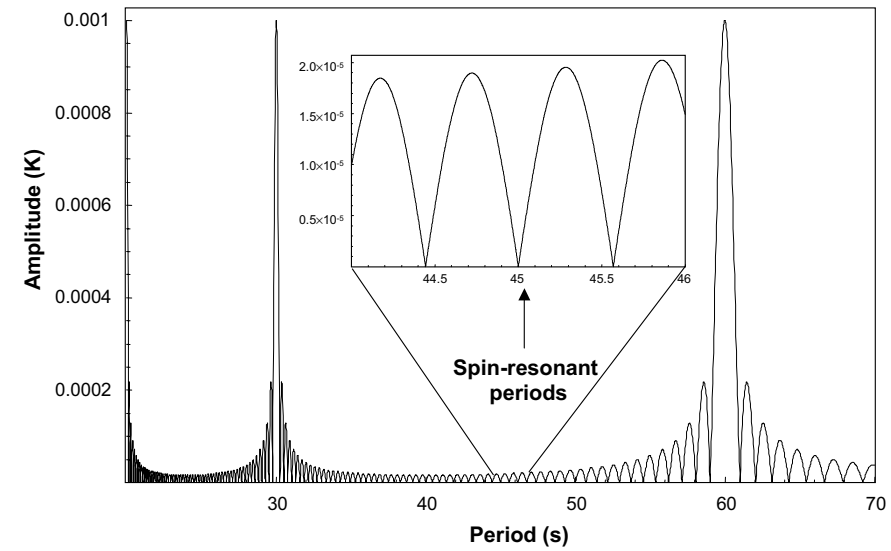

Fig. 1. Plot of $\left\langle\delta T_{\text {sky }}^{\mathrm{p}-\mathrm{p}}\right\rangle_{N}$ for for $A_{\mathrm{f}}=0.5 \mathrm{mK}$ and $\tau_{\mathrm{f}}$ between $20 \mathrm{~s}$ and $70 \mathrm{~s}$. The graph shows the two peaks relative to the spin synchronous periods of $60 \mathrm{~s}$ and $30 \mathrm{~s}$ (for which $\left\langle\delta T_{\text {sky }}^{\mathrm{p}-\mathrm{p}}\right\rangle_{N}=2 A_{\mathrm{f}}$ ) and a series of spin resonant periods (for which $\left\langle\delta T_{\text {sky }}^{\mathrm{p}-\mathrm{p}}\right\rangle_{N}=0$, see inset).

data stream after averaging over $N$ scan circles is simply given by:

$\left\langle\delta T_{\text {sky }}^{\mathrm{p}-\mathrm{p}}\right\rangle_{N}=2\left|\frac{A_{\mathrm{f}}}{N} \frac{\sin \left(\pi N \tau_{\text {spin }} / \tau_{\mathrm{f}}\right)}{\sin \left(\pi \tau_{\text {spin }} / \tau_{\mathrm{f}}\right)}\right|$.

Two special cases can be derived from the above equation: the case of "spin synchronous" fluctuations (for which $\tau_{\mathrm{f}}=\tau_{\text {spin }} / k$ where $k$ is any integer) and the case of "spin resonant" fluctuations (for which $\tau_{\mathrm{f}}=N \tau_{\text {spin }} / k$, where $k$ is any integer not multiple of $N$ ).

Spin synchronous fluctuations are not damped by the measurement redundancy ${ }^{1}$ while spin resonant fluctuations, instead, are such that for every pixel the average fluctuation after $N$ consecutive measurements is zero.

Let us now consider first oscillations with $\tau_{\mathrm{f}} \leq \tau_{\text {spin }}$. In Fig. 1 we show a plot of $\left\langle\delta T_{\text {sky }}^{\mathrm{p}-\mathrm{p}}\right\rangle_{N}$ given by Eq. (3) for values of $\tau_{\mathrm{f}}$ up to $70 \mathrm{~s}$, considering PLANCK nominal values $\left(\tau_{\text {spin }}=60 \mathrm{~s}\right.$ and $\left.N=60\right)$ and assuming $A_{\mathrm{f}}=0.5 \mathrm{mK}$.

The picture shows that the residual peak-to-peak fluctuation in a sky scan circle equals $2 A_{\mathrm{f}}$ for spinsynchronous periods and is of the order of $2 A_{\mathrm{f}} / N$ otherwise. The inset in Fig. 1 highlights that for spin resonant periods the oscillation is completely cancelled by the averaging.

Figure 2 shows the case of oscillations with $\tau_{\mathrm{f}}>\tau_{\text {spin }}$. In this case there are no more spin synchronous periods, but we still have an effect of resonant periods up to $\tau_{\mathrm{f}}=N \times \tau_{\text {spin }}$ (which equals $3600 \mathrm{~s}$ in our model). The envelope defining the upper limit of $\left\langle\delta T_{\text {sky }}^{\mathrm{p}-\mathrm{p}}\right\rangle_{N}$ (solid line) is given by:

$$
\left\langle\delta T_{\text {sky }}^{\mathrm{p}-\mathrm{p}}\right\rangle_{N}^{\max }=2\left|\frac{A_{\mathrm{f}}}{N} \frac{1}{\sin \left(\pi \tau_{\text {spin }} / \tau_{\mathrm{f}}\right)}\right| .
$$

Some remarks need to be made concerning the relevance of spin-resonant and spin-synchronous periods in the context of PLANCK measurements.

1 This implication can be easily derived from Eq. (3) considering that $\lim _{x \rightarrow 1}(\sin (N \pi x) / \sin (\pi x))=N$. 


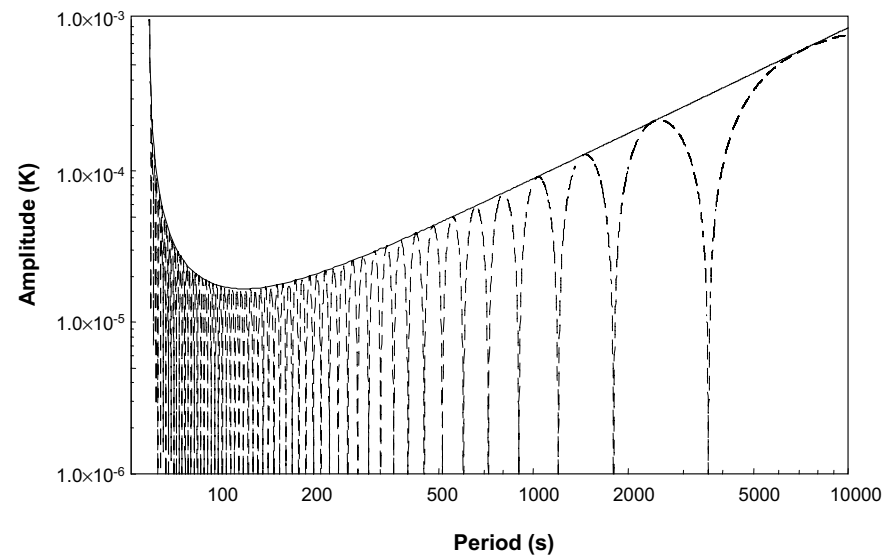

Fig. 2. Plot of $\left\langle\delta T_{\text {sky }}^{\mathrm{p}-\mathrm{p}}\right\rangle_{N}$ for for $A_{\mathrm{f}}=0.5 \mathrm{mK}$ and $\tau_{\mathrm{f}}$ between $60 \mathrm{~s}$ and $10000 \mathrm{~s}$. The graph shows that no more spin synchronous periods exist after $\tau_{\mathrm{f}}=60 \mathrm{~s}$, while the last spin resonant period is at $\tau_{\mathrm{f}}=3600 \mathrm{~s}$. The solid line represents the envelope defined by Eq. (4).

Spin-resonant fluctuations in principle do not contribute to the overall effect; in reality many non-ideal behaviours (like instability in the spin period, pointing errors, variations in the repointing time, etc.) will concur in eliminating such sharp resonances. The impact of spinsynchronous periods (in particular $\tau_{\mathrm{f}}=60 \mathrm{~s}$ ), instead, will be less sensitive to smearing by scan non idealities. In fact if we consider the main peak in Fig. 1 we see that it has a width (at half maximum) of $\sim 1 \mathrm{~s}$. Because the PLANCK spin rate will be stable at the level of $\sim 10^{-4} \mathrm{rpm} / \mathrm{h}$ it follows that even in the case of a constant drift in the spin rate a fluctuation which is initially spin-synchronous will maintain this characteristic for time-scales of the order of one week.

Therefore, in our paper we will adopt a conservative approach by neglecting only the effect of spin-resonant periods. This implies that Eq. (4) is used to estimate the effect on the measured data stream of fluctuations with $\tau_{\mathrm{f}}>\tau_{\text {spin }}$.

\subsection{Level of residual fluctuations in final maps}

The simplest way to estimate the impact of periodic fluctuations on the final measurements is to project the data stream of measurements averaged over multiple scans onto a map with a given pixel size and then evaluate the peakto-peak amplitude. This rather simple procedure gives a gross upper limit of the amplitude of the effect, which is usually greatly reduced by applying so-called "destriping" algorithms to the time ordered data.

In the following we derive a general relationship to estimate the final peak-to-peak amplitude of a given systematic effect by knowing its amplitude at the instrument output, its frequency and the map pixel size. This relationship is obtained analysing the simple projection of the periodic signal onto the sky map and then considering the additional damping factor obtained after the application of our destriping code to the data stream.

\subsubsection{Projection of averaged data streams onto sky maps}

The projection of a time ordered array of data onto a map consists in the association of every data sample to a pixel coordinate in the sky.

If we consider the PLANCK scanning strategy it is clear that pixels close to the ecliptic plane will be "visited" by a smaller number of scan circles compared to pixels around the ecliptic poles. Therefore, the amplitude of a fluctuation projected onto a sky map will be maximum along the ecliptic, where each pixel is crossed by $N_{\text {scan }} \approx$ $\theta_{\text {pixel }} / \theta_{\text {rep }}$ circles, where $\theta_{\text {pixel }}$ is the pixel size and $\theta_{\text {rep }}$ indicates the repointing angle, that in PLANCK current baseline is $\sim 2.5^{\prime}$.

A rather simple analysis of the data averaging in pixels around the ecliptic plane leads to the following formula for the peak-to-peak amplitude of a fluctuation with amplitude $A_{\mathrm{f}}$ and period $\tau_{\mathrm{f}}$ when projected onto a sky map with pixel size $\theta_{\text {pixel }}$ :

$\left\langle\delta T_{\text {sky }}^{\mathrm{p}-\mathrm{p}}\right\rangle_{\text {map }}=2 \frac{A_{\mathrm{f}}}{N \times N_{\text {scan }}}\left|\frac{\sin \left(\pi N \times N_{\text {scan }} \tau_{\text {spin }} / \tau_{\mathrm{f}}\right)}{\sin \left(\pi \tau_{\text {spin }} / \tau_{\mathrm{f}}\right)}\right|$.

From Eq. (5) it appears that $\left\langle\delta T_{\text {sky }}^{\mathrm{p}-\mathrm{p}}\right\rangle_{\text {map }}$ can be zero for every value of $\tau_{\mathrm{f}}$ that satisfies the relationship $\tau_{\mathrm{f}}=N \times$ $N_{\text {scan }} \tau_{\text {spin }} / k$, with $k$ integer. A set of these values is what we have called spin resonant periods, i.e. those periods which are resonant with the scan circle time $N \times \tau_{\text {spin }}$. Another set of these values corresponds to periods that are resonant with the repointing time $\tau_{\text {scan }}=N_{\text {scan }} \times \tau_{\text {spin }}$. Considering that $N_{\text {scan }}$ is not constant all over the map (pixels close to the ecliptic poles are crossed by a greater number of scans compared to pixels close to the ecliptic plane) this latter set of periods does not actually produce a global zero-effect, but only in a limited number of pixels.

Therefore, with the conservative assumption that spin resonant periods will not be relevant in practice, we can conclude that the best estimate of the peak-to-peak amplitude $\left\langle\delta T_{\text {sky }}^{\mathrm{p}-\mathrm{p}}\right\rangle_{\text {map }}$ can be well approximated by the upper limit of Eq. (5), i.e.:

$$
\begin{aligned}
& \left\langle\delta T_{\text {sky }}^{\mathrm{p}-\mathrm{p}}\right\rangle_{\text {map }} \approx\left\langle\delta T_{\text {sky }}^{\mathrm{p}-\mathrm{p}}\right\rangle_{\text {max }} \text { max } \\
& =2 \frac{A_{\mathrm{f}}}{N \times N_{\text {scan }}}\left|\frac{1}{\sin \left(\pi \tau_{\text {spin }} / \tau_{\mathrm{f}}\right)}\right|=\frac{2 A_{\mathrm{f}}}{F_{\text {map }}}
\end{aligned}
$$

where $F_{\text {map }}$ is the damping factor of the oscillation from the instrument output to the sky map.

We can now compare the damping factors calculated by Eq. (6) with those obtained by projecting periodic signals with different values of $\tau_{\mathrm{f}}$ onto sky maps. The maps (produced according to the HEALPix hierarchical structure, Gòrski et al. 1998) are relative to the position of a $30 \mathrm{GHz}$ LFI radiometer and have a pixel size of $13.7^{\prime}$. All the maps have been produced assuming 10200 hours observation and the PLANCK baseline scanning strategy, which implies an average integration time per pixel of $\sim 46.7$ s. 


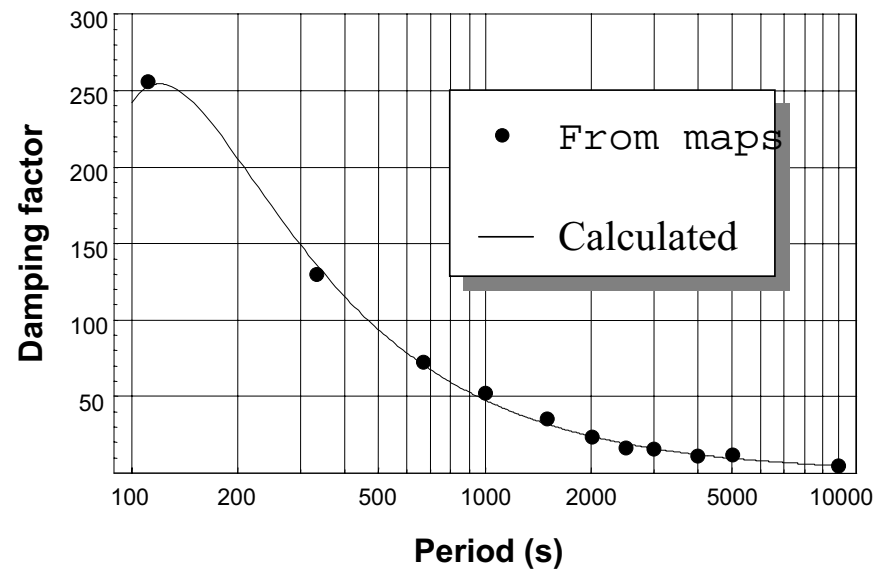

Fig. 3. Comparison of the damping factor as a function of $\tau_{\text {f }}$ calculated by Eq. (6) (solid line) and obtained from maps (black dots) $\left(\theta_{\text {pixel }}=13.7^{\prime}, \theta_{\text {scan }}=2.5^{\prime}\right)$.

Figure 3 shows the result of this comparison and highlights a remarkable agreement between the damping factors obtained directly from maps and calculated from Eq. (6).

\subsubsection{Removal of periodic systematic effects from time ordered data}

In this section we evaluate the residual systematic error on the maps after the application of a "destriping" code developed by the LFI team for reducing the impact of $1 / f$ noise fluctuations.

This code generally uses averaged one-hour scan circles (although it has the capability to work with the full, unaveraged data streams); because averaging acts like a low-pass filter, only the very low frequencies remain in the map as a residual effect. The basic hypothesis is that for each circle the residual effect can be well approximated by a constant additive level related to the mean level of the spurious fluctuation during the scan. The code optimises these levels by minimising the map temperature values in pixels observed by more than one scan circle during the mission. Further details of the code implementation can be found in Burigana et al. (1997b).

In Fig. 4 we show an example of the application of this code to a pure periodic fluctuation with a period of $4000 \mathrm{~s}$ and an amplitude (at the instrument output) of $1 \mathrm{mK}$ peak-to-peak. A comparison between the two maps shows that the code is able to reduce the impact of such fluctuation by a factor of $\sim 34$.

So far we have considered the application of the destriping code to time ordered data containing only the periodic fluctuation, i.e. with no noise present. We have also shown that the presence of white and $1 / f$ noise in the data stream does not change the ability of the code to remove periodic fluctuations. To verify this we have applied our code to a data stream containing noise (white and $1 / f)$ plus a periodic signal and to a second data stream containing only white and $1 / f$ noise.
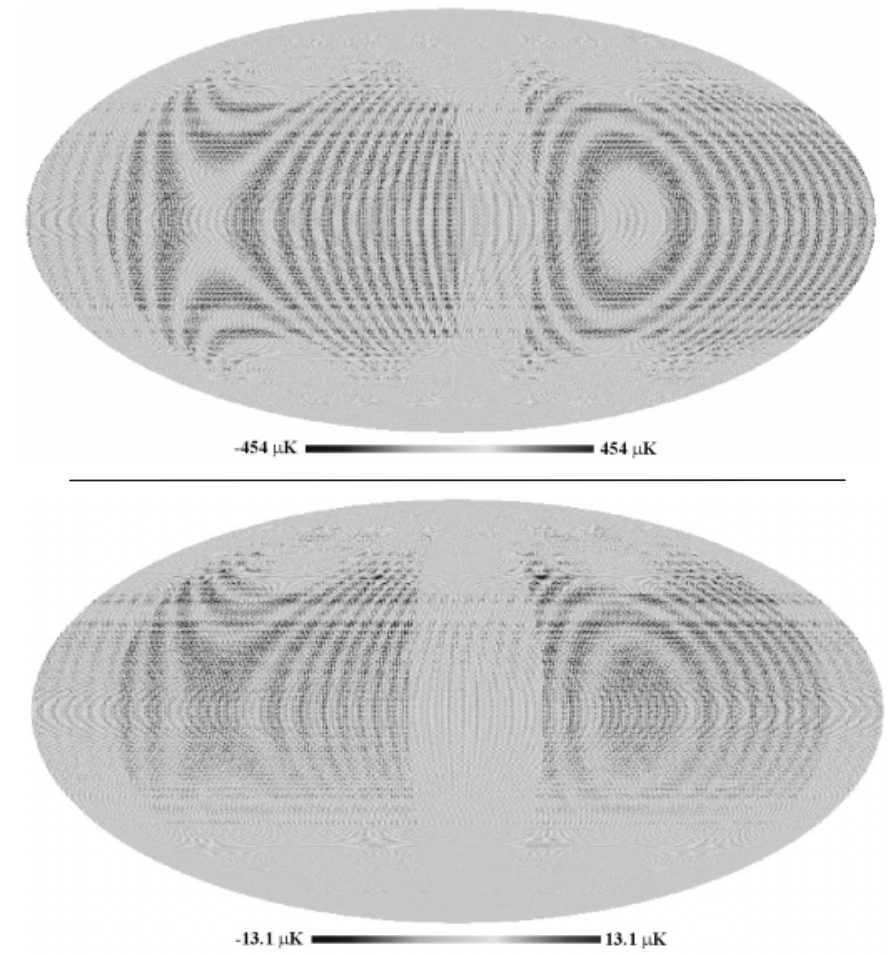

Fig. 4. Application of the destriping code to a "pure" periodic systematic effect. The upper map is relative to a sinusoidal fluctuation with a $\tau_{\mathrm{f}}=4000 \mathrm{~s}$ and $A_{\mathrm{f}}=5 \mathrm{mK}$. After postprocessing the time ordered data with our destriping code the peak-to-peak effect on the map is reduced by a factor $\sim 34$.

The noise is typical of LFI $30 \mathrm{GHz}$ radiometers, i.e. white noise and $1 / f$ noise, with a spectral density of $230 \mu \mathrm{K} \mathrm{Hz}^{-1 / 2}$ and a knee frequency of $0.1 \mathrm{~Hz}$ (rather conservative estimates of the expected performances $30 \mathrm{GHz}$ LFI radiometers $)^{2}$. The periodic fluctuation is the same as in Fig. 4.

By taking the difference between the map containing noise plus the periodic fluctuation and the map containing only noise (both after destriping) we obtain a map that is virtually indistinguishable (at the level of $10^{-5} \mu \mathrm{K}$ ) from the bottom map of Fig. 4, which was obtained by applying the destriping algorithm to the periodic oscillation without noise. This indicates that the algorithm was able to recognise and reduce the effect of the periodic fluctuation even in presence of noise stream with a much larger amplitude level.

Our next step has been to evaluate the destriping damping factor for periodic fluctuations as a function of $\tau_{\mathrm{f}}$. Figure 5 shows how this damping factor increases approximately linearly with $\tau_{\text {f }}$ for periods greater than the spin period $^{3}$. It is important to underline that the exact form of the linear interpolation shown in Fig. 5 is dependent on

\footnotetext{
${ }^{2}$ The reported spectral density is relative to the noise requirement of the $30 \mathrm{GHz}$ LFI radiometers and it has been chosen as a conservative case. The goal value is of about $180 \mu \mathrm{K} \cdot \mathrm{Hz}^{-1 / 2}$ with an expected knee frequency of $0.05 \mathrm{~Hz}$.

${ }^{3}$ For spin-synchronous fluctuations no damping of the effect is obtained from the application of destriping codes.
} 


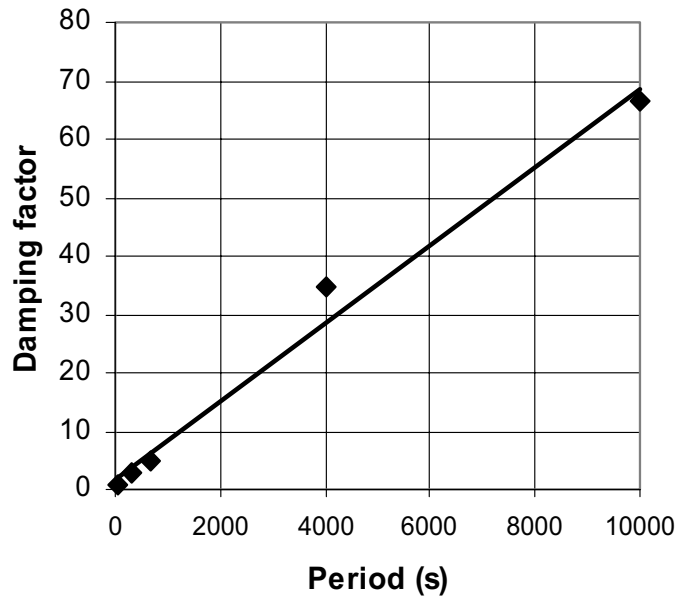

Fig. 5. Destriping damping factor for periodic oscillations ver$\operatorname{sus} \tau_{\mathrm{f}}$. Dots indicate the values obtained from maps while the solid line represents the linear interpolation $F_{\text {destr }}\left(\tau_{\mathrm{f}}\right)=a \tau_{\mathrm{f}}+b$ with $a=0.1067 \mathrm{~s}^{-1}$ and $b=1.7992$.

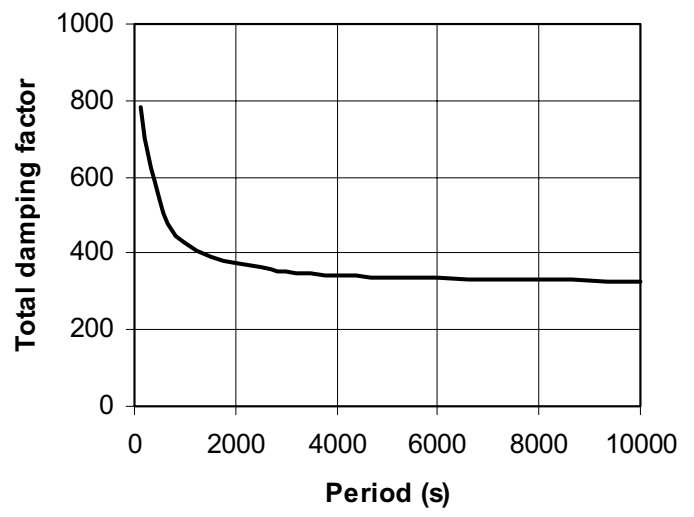

Fig. 6. Total damping factor from instrument output to final map for the peak-to-peak amplitude of periodic oscillations having a period $\tau_{\mathrm{f}}>\tau_{\text {spin }}$ considering the destriping damping as in Fig. 5.

the conditions under which the maps have been calculated, which are, in the case discussed here, a sampling rate of 1 sample every $\sim 12^{\prime}$ and a map pixel size of $13.7^{\prime}$ (typical conditions of LFI $30 \mathrm{GHz}$ channels).

If we indicate with $F_{\text {destr }}\left(\tau_{\mathrm{f}}\right)=a \tau_{\mathrm{f}}+b$ the destriping damping factor, we can write the following general form for the total damping factor of the peak-to-peak amplitude of periodic oscillations from instrument output to final map:

$F\left(\tau_{\mathrm{f}}\right) \geq F_{\text {map }}\left(\tau_{\mathrm{f}}\right) \times F_{\text {destr }}\left(\tau_{\mathrm{f}}\right)$

where $F_{\text {map }}$ can be calculated by Eq. (6). Note that Eq. (7) provides a lower limit of the total damping factor rather than the exact value. This is a consequence of our assumptions regarding the beam position and the scanning strategy which are marginally conservative with respect to the destriping efficiency.

In Fig. 6 we show a plot of the function $F\left(\tau_{\mathrm{f}}\right)$ considering the destriping damping factor relative to the case shown in Fig. 5.

\section{Application to a periodic fluctuation of arbitrary shape}

In this section we extend our analysis to periodic fluctuations of general shape and show some examples of its application. Under very general assumptions we can write the oscillation in Fourier series as:

$\delta T_{\mathrm{sky}}(t)=\sum_{j=-\infty}^{+\infty} A_{j} \exp \left(i 2 \pi \nu_{j} t\right)$

where $\nu_{j}$ represent the harmonic frequencies. We now apply the same procedure discussed in the previous sections to each term of the series in Eq. (8) in order to calculate the peak-to-peak amplitude after one-hour averaging and after the projection of the averaged time data stream onto a map. Considering that for each harmonic term we have:

$\sum_{k=1}^{N} A_{j} \exp \left(i 2 \pi \nu_{j}\left(t_{0}+(k-1) \tau_{\text {spin }}\right)\right)$

$=A_{j} \exp \left[i \pi\left(2 t_{0}+(N-1) \tau_{\mathrm{spin}}\right) \nu_{j}\right] \frac{\sin \left(N \pi \tau_{\mathrm{spin}} \nu_{j}\right)}{\sin \left(\pi \tau_{\mathrm{spin}} \nu_{j}\right)}$

then the final peak-to-peak amplitude for a general signal on the map after destriping can be written as:

$\left\langle\delta T_{\text {sky }}^{\mathrm{p}-\mathrm{p}}\right\rangle_{\text {max }}^{\max }=\frac{2}{N \times N_{\text {scan }}}\left|\sum_{j=-\infty}^{+\infty} \frac{A_{j} / F_{\text {destr }}\left(\nu_{j}\right)}{\sin \left(\pi \tau_{\text {spin }} \nu_{j}\right)}\right|$.

Now we show an example of the application of this formalism to the signal oscillation shown in Fig. 7 both in time domain (upper graph) and Fourier space (lower graph) ${ }^{4}$.

This signal oscillation represents an estimate of the thermally-induced signal instability caused by the PLANCK Sorption Cooler working with degraded performances. It is worth underlying that this estimate was derived from simulations ${ }^{5}$ which do not represent a quantitative prediction of the expected Sorption Cooler performances, but were aimed at understanding qualitatively the impact of compressor non-idealities on the temperature stability. This oscillation pattern is used in our paper merely as an example of the application of our analysis to signal oscillations of complex shape.

From the figure it is apparent that the fluctuation is dominated by two main periods (667 s and $4000 \mathrm{~s}$ ) but it also contains a high number of harmonics with periods down to about $40 \mathrm{~s}$. The inset in the lower graph of Fig. 7 is a close-up of the high frequency tail of the fluctuation spectrum.

By applying Eq. (10) to the Fourier transform of the signal we are able to calculate the expected peak to peak amplitude of this effect when it is processed by our destriping code and projected onto a $13.7^{\prime}$ map. In the case discussed here the computation yields an expected value

\footnotetext{
${ }^{4}$ Note that in the abscissa axis of the Fourier transform we indicate the period rather than the frequency.

${ }^{5}$ Simulations were run using the Sorption Cooler thermal model implemented with the SINDA simulation software.
} 

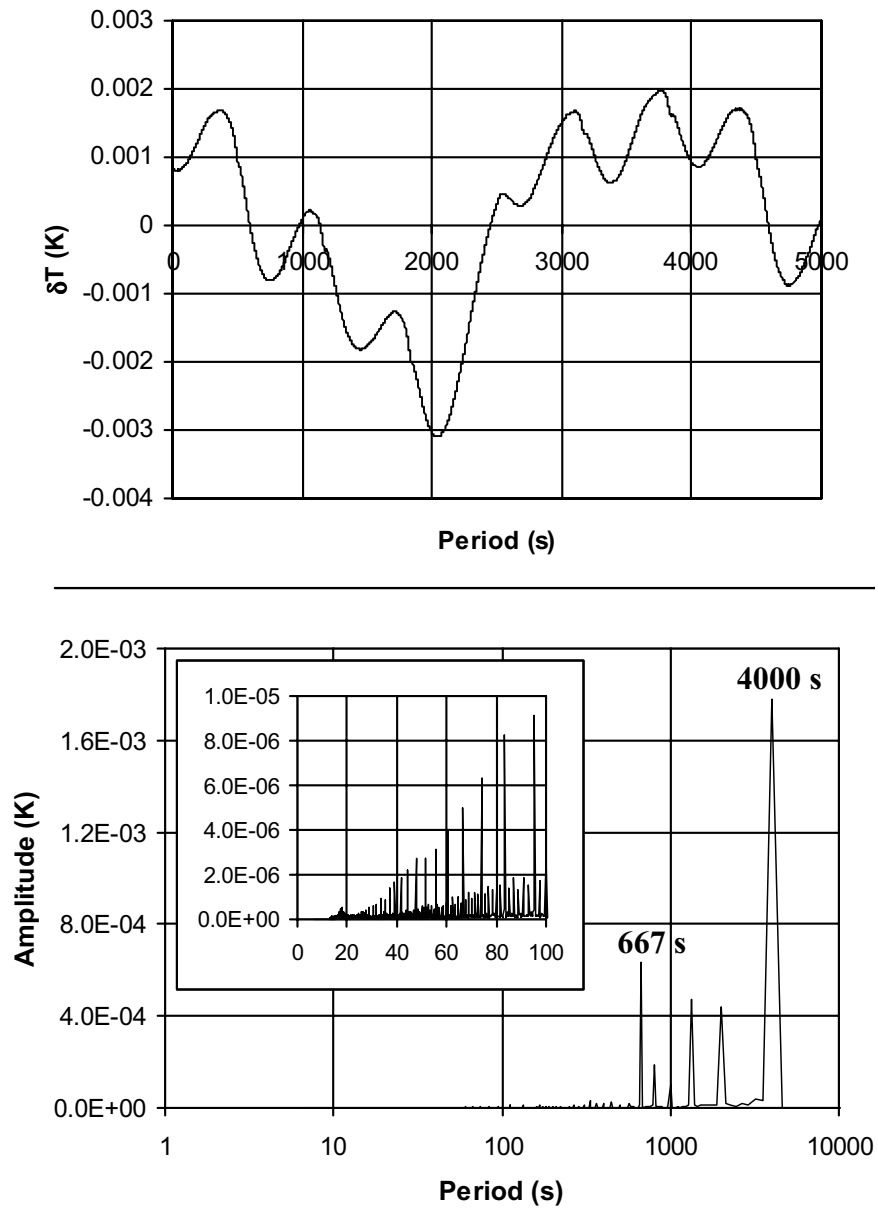

Fig. 7. Signal oscillation in time domain (upper graph) and Fourier space (lower graph) representing a simulation of the thermally-induced signal instability caused by a Sorption Cooler working with degraded performances. The inset in the lower graph is a close-up of the high frequency tail of the fluctuation spectrum.

of $13.37 \mu \mathrm{K}$ for the final peak-to-peak amplitude, which is in excellent agreement with the value obtained by the standard mapping procedure (see Fig. 8).

Note that in Fig. 8 the morphology of the residual systematic on the map (which is relative to the signal oscillation shown in Fig. 7) is very similar to the morphology shown in the bottom map of Fig. 4 (relative to a "pure" periodic signal with $\tau_{\mathrm{f}}=4000 \mathrm{~s}$ ). This is the consequence of the fact that the oscillation in Fig. 7 is dominated by the $4000 \mathrm{~s}$ wave (as shown in the Fourier transform) which determines the main visible structure in the map.

The same procedure was also applied to other two signal fluctuations with very different shapes, which represent thermally-induced fluctuations for various Sorption Cooler performance scenarios. The results of the three tests summarised in Table 1 show a very good agreement between the final peak-to-peak amplitudes obtained directly from maps and calculated with Eq. (10), which confirms the general validity of our approach.

The values reported in the last two columns of Table 1 are relative to $30 \mathrm{GHz}$ LFI maps with a pixel size

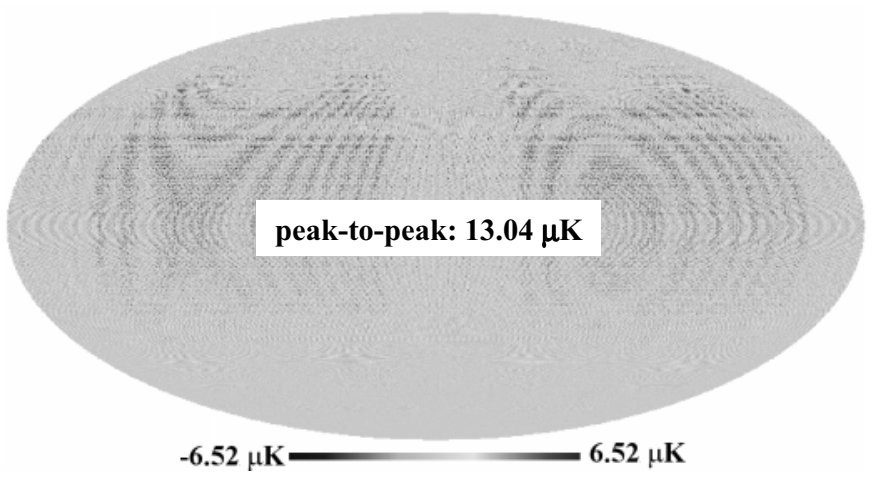

Fig. 8. Map (pixel size $=13.7^{\prime}$ ) after destriping of the signal fluctuation plotted in Fig. 7.

$\theta_{\text {pixel }}=13.7^{\prime}$. Because the optical beam size will be approximately three times larger than $\theta_{\text {pixel }}$, the actual amplitude of the systematic effect on the instrument angular scale would be much smaller, ranging from $\sim 0.5 \mu \mathrm{K}$ to $\sim 5 \mu \mathrm{K}$ for the three cases reported in Table 1 .

Although further analysis (which is out of the scope of our paper) is needed to understand the impact on the recovered amplitude and polarisation power spectra, our analysis can give rather precise estimates of the damping factor of the peak-to-peak amplitude of the fluctuation at the instrument output after the projection in the final maps.

\section{Conclusions}

In this paper we have proposed an analytic method to estimate the effect of periodic signal oscillations on CMB maps to be produced by sky scanning experiments. When these signal fluctuations are processed with a destriping algorithm and subsequently projected onto a sky map, the amplitude is reduced by a factor of several hundreds; an exception is represented by spin-synchronous oscillations that, in general, are not damped by the scanning strategy and can be reduced by dedicated algorithms only in special cases where we have information about the behaviour of these signals (e.g. external stray light, Delabrouille et al. 2001).

We have derived an analytical transfer function of the peak-to-peak amplitude from the signal oscillation at the instrument output to the map before destriping. The main result is a general relationship that allows to estimate peak-to-peak effect of arbitrary periodic fluctuations on maps with arbitrary pixel size.

The additional damping obtained by applying a destriping algorithm has been derived as a function of the oscillation period for maps that are typical of the $30 \mathrm{GHz}$ PLANCK-LFI channels. The application of this procedure to a signal fluctuation of complex shape has shown that 
Table 1. Application of our analytical procedure to signal fluctuations of different shape and amplitude. These fluctuations represent the effect of the Sorption Cooler working with different levels of performance.

\begin{tabular}{|c|c|c|c|c|}
\multicolumn{2}{c|}{} & $\begin{array}{c}\text { Peak-to-peak amplitude on } \\
\text { final maps after destriping } \\
\text { with 13.7' pixel size } \\
(\mu \mathrm{K})\end{array}$ \\
\hline $\begin{array}{c}\text { Shape of } \\
\text { signal } \\
\text { fluctuation }\end{array}$ & $\begin{array}{c}\text { Sorption } \\
\text { Cooler } \\
\text { performance }\end{array}$ & $\begin{array}{c}\text { Peak-to- } \\
\text { peak } \\
\text { amplitude } \\
(\mathrm{mK})\end{array}$ & $\begin{array}{c}\text { From } \\
\text { analytical } \\
\text { calculation }\end{array}$ & $\begin{array}{c}\text { From } \\
\text { map }\end{array}$ \\
\hline & Nominal & 1.06 & 2.84 & 2.31 \\
\hline & Degraded & $\mathbf{4 . 3 0}$ & 13.37 & 13.04 \\
\hline & Highly & $\mathbf{8 . 2 4}$ & $\mathbf{2 2 . 8 8}$ & $\mathbf{2 1 . 6 5}$ \\
\hline
\end{tabular}

it is possible to predict accurately the final peak-to-peak effect.

Although this study has been performed in the context of PLANCK measurements, the results obtained are generally applicable to any sky imaging experiment involving redundant pixel measurements. Further developments of this study will be aimed at applying these results to predict the impact of different kinds of periodic systematic effects in PLANCK-LFI.

Acknowledgements. Mauro Prina (Jet Propulsion Laboratory, Pasadena, USA) contributed in simulating the Sorption Cooler temperature stability characteristics, Roberto Ferretti (LABEN S.p.A., Milano, Italy) contributed in simulating the thermal behaviour of the PLANCK-LFI instrument. Both contributions have been fundamental to define the signal oscillations at the basis of the results reported in Table 1.

The HEALPix package use is acknowledged (see HEALPix home page at http://www.eso.org/science/healpix/). We also wish to thank the Planck LFI Data Processing Center for the support to the simulation work.

\section{References}

Bersanelli, M., \& Mandolesi, N. 2000, Astroph. Lett. \& Comm., 37,171

Bhandari, P., Prina, M., Ahart, M., et al. 2000, in Proc. 11th Int. Cryocooler Conf., 541

Bond, J. R., \& Efstathiou, G. 1987, MNRAS, 226, 407

Burigana, C., Seiffert, M., Mandolesi, N., et al. 1997, Internal Report ITeSRE 186/1997

Burigana, C., Malaspina, M., Mandolesi, N., et al. 1997, Internal Report ITeSRE 198/1997 [astro-ph/9906360]

Burigana, C., Maino, D., Mandolesi, N., et al. 1998, A\&AS, 130,551

Burigana, C., Maino, D., Gòrski, K. M., et al. 2000, A\&A, 373, 345

Danese, L., Toffolatti, L., Franceschini, A., et al. 1996, Astroph. Lett. \& Comm., 33, 257

De Bernardis, P., Ade, P. A. R., Bock, J. J., et al. 2000, Nature, 404, 955

Delabrouille, J., Patanchon, G., \& Audit, G. 2001 [astro-ph/0109187]

Gòrski, K. M., Hivon, E., \& Wandelt, B. D. 1998, Proc. MPA/ESO Conference on Evolution of Large-Scale Structure: from Recombination to Garching, ed. A. J. Banday, R. K. Sheth, \& L. Da Costa, 37

Halverson, N. W., Leitch, E. M., Pryke, C., et al. 2001, ApJ, submitted [astro-ph/0104489]

Lee, A. T., Ade, P., Balbi, A., et al. 2001, ApJL, submitted [astro-ph/0104459]

Maino, D., Burigana, C., Maltoni, M., et al. 1999, A\&AS, 140, 383

Mandolesi, N., Bersanelli, M., Burigana, C., et al. 2000, A\&AS, 145,323

Mandolesi, N., Bersanelli, M., Burigana, C., et al. 2000, Astroph. Lett. Comm., 37, 151 [astro-ph/9904135]

Mather, J. C., Fixsen, D. J., Shafer, R. A., et al. 1999, ApJ, 512,511

Netterfield, C. B., Ade, P. A. R., Bock, J. J., et al. 2001, ApJ, submitted [astro-ph/0104460]

Prina, M., Bhandari, P., Bowman, R. C., et al. 2001, to be published in Adv. in Cryogenic Eng.

Pryke, C., Halverson, N. W., Leitch, E. M., et al. 2001, ApJ, submitted [astro-ph/0104490]

Seiffert, M., Mennella, A., Burigana, C., et al. 2001, Rev. Sci. Inst., submitted

Smoot, G. F., et al. 1992, ApJ, 396, L1

Stompor, R., Abroe, M., Ade, P., et al. 2001, ApJL, submitted [astro-ph/0105062]

Vittorio, N., \& Silk, J. 1984, ApJ, 285, 39 